\title{
Spectral Regularization Methods for an Abstract Ill-Posed Elliptic Problem
}

\author{
Nadjib Boussetila, ${ }^{1,2}$ Salim Hamida, ${ }^{2}$ and Faouzia Rebbani ${ }^{2}$ \\ ${ }^{1}$ Department of Mathematics, University 8 Mai 1945 Guelma, P.O. Box 401, 24000 Guelma, Algeria \\ ${ }^{2}$ Applied Mathematics Laboratory, University Badji Mokhtar Annaba, P.O. Box 12, 23000 Annaba, Algeria \\ Correspondence should be addressed to Nadjib Boussetila; n.boussetila@gmail.com
}

Received 10 April 2013; Accepted 28 September 2013

Academic Editor: Alberto Fiorenza

Copyright (c) 2013 Nadjib Boussetila et al. This is an open access article distributed under the Creative Commons Attribution License, which permits unrestricted use, distribution, and reproduction in any medium, provided the original work is properly cited.

We study an abstract elliptic Cauchy problem associated with an unbounded self-adjoint positive operator which has a continuous spectrum. It is well-known that such a problem is severely ill-posed; that is, the solution does not depend continuously on the Cauchy data. We propose two spectral regularization methods to construct an approximate stable solution to our original problem. Finally, some other convergence results including some explicit convergence rates are also established under a priori bound assumptions on the exact solution.

\section{Introduction}

Throughout this paper $H$ denotes a complex Hilbert space endowed with the inner product $(\cdot, \cdot)$, and the norm $\|\cdot\|, \mathscr{L}(H)$ stands for the Banach algebra of bounded linear operators on $H$.

Let $A$ be a linear unbounded operator with dense domain $D(A)$. Assume that $A$ is self-adjoint, positive definite in $H$, which has a continuous spectrum $\sigma(A)=[\gamma,+\infty[, \gamma=$ $\inf (\sigma(A))>0$.

We consider the elliptic Cauchy problem (ECP) of finding $u:[0, L] \rightarrow H$ such that

$$
\begin{gathered}
u_{y y}(y)=A u(y), \quad 0<y<L, \\
u(0)=f, \\
u_{y}(0)=0,
\end{gathered}
$$

where $f$ is some prescribed data in the Hilbert space $H$.

This problem is an abstract version of Cauchy problem, which generalizes Cauchy problem for second-order elliptic partial differential equations in a cylindrical domain; for example, we mention the following problem.
Example 1. An example of (1) is the Cauchy problem for the modified Helmholtz equation in the infinite strip $\mathbb{R} \times(0,1)$ [1]:

$$
\begin{array}{r}
u_{y y}(x, y)+u_{x x}(x, y)-\gamma u(x, y)=0, \\
x \in \mathbb{R}, \quad y \in(0, L), \\
u(x, 0)=f(x), \quad u_{y}(x, 0)=0, \quad x \in \mathbb{R},
\end{array}
$$

where the operator $A_{\gamma}$ is given by

$$
\begin{gathered}
A_{\gamma}=-\frac{\partial^{2}}{\partial x^{2}}+\gamma I, \\
D\left(A_{\gamma}\right)=H^{2}(\mathbb{R}) \subset H=L^{2}(\mathbb{R}) .
\end{gathered}
$$

It is well known that this operator is self-adjoint with continuous spectrum

$$
\sigma\left(A_{\gamma}\right)=\sigma\left(A_{0}\right)+\gamma=[0,+\infty[+\gamma=[\gamma,+\infty[.
$$

We note here that the discrete eigenfunctions expansion method cannot be used, but we can use the Fourier diagonalization method to deal with this kind of problems. 
Such problem arises in many practical situations, nondestructive testing techniques [2], geophysics [3], cardiology [4], and other applications. There are many various monographs about the historical development of this topic, for more details, we refer the reader to $[5,6]$. Recently there has been an excellent topic review [7] of this problem.

Because problem (1) is severely ill-posed; that is, a small perturbation in the given Cauchy data may result in a very large error on the solution. In order to overcome this instability character, the regularization methods are required.

Some regularization methods for the Cauchy problem for elliptic equations have been proposed by many authors. For instance: Tikhonov regularization method [8], the quasireversibility method [9], the quasi-boundary-value method [10-13], Kozlov-Maz'ya iteration method [14], and the mollification method [15].

This work is mainly devoted to theoretical aspects of the spectral regularization methods to problem (1) in the abstract setting, by considering more general self-adjoint operators when $A$ is positive and induces the elliptic case, that is, has the following properties: for any $\lambda \in(-\infty, 0]$, the resolvent $R(\lambda ; A)=(A-\lambda I)^{-1}$ exists and satisfies the estimates

$$
\exists M>0: \forall \lambda \geq 0, \quad\left\|(A+\lambda I)^{-1}\right\| \leq M(1+\lambda)^{-1} .
$$

In the case when $A$ is a linear positive self-adjoint operator with compact inverse, problem (1) has been treated by a different method and there is a large literature in this direction. However, in the case where $A$ has a continuous spectrum the literatures are quite scarce.

In the present paper we shall use two spectral regularization methods to construct a stable solution to our original illposed problem.

\section{Preliminaries and Basic Results}

In this section we present the notation and the functional setting which will be used in this paper and prepare some material which will be used in our analysis.

2.1. Spectral Theorem and Properties. By the spectral theorem, for each positive self-adjoint operator $A$, there is a unique right continuous family $\left\{E_{\lambda}\right\}_{\lambda \in[0, \infty[}:[0, \infty[\rightarrow \mathscr{L}(H)$ of orthogonal projection operators such that $A=\int_{0}^{\infty} \lambda d E_{\lambda}$ with

$$
D(A)=\left\{v \in H: \int_{0}^{\infty} \lambda^{2} d\left(E_{\lambda} v, v\right)<\infty\right\} .
$$

In our case, we have $A=\int_{\gamma}^{\infty} \lambda d E_{\lambda}$ because $A \geq \gamma I, \gamma>0$.

Theorem 2 (see [16, Theorem 6, XII.2.5, pages 1196-1198]). Let $\left\{E_{\lambda}, \lambda \geq \gamma>0\right\}$ be the spectral resolution of the identity associate to $A$ and let $\phi$ be a complex Borel function defined E-almost everywhere on the real axis. Then $\phi(A)$ is a closed operator with dense domain. Moreover

(i) $D(\phi(A)):=\left\{v \in H: \int_{\gamma}^{\infty}|\phi(\lambda)|^{2} d\left(E_{\lambda} v, v\right)<\infty\right\}$,

(ii) $(\phi(A) h, y)=\int_{\gamma}^{\infty} \phi(\lambda) d\left(E_{\lambda} h, y\right), h \in D(\phi(A)), y \in H$, (iii) $\|\phi(A) h\|^{2}=\int_{\gamma}^{\infty}|\phi(\lambda)|^{2} d\left(E_{\lambda} h, h\right), h \in D(\phi(A))$,

(iv) $\phi(A)^{*}=\bar{\phi}(A)$. In particular, if $\phi$ is real Borel function, then $\phi(A)$ is self-adjoint,

(v) the operator $\phi(A)$ is bounded if and only if $\phi(\lambda)$ is bounded on $\sigma(A)=[\gamma,+\infty[$. In this case, $\|\phi(A)\|=$ $\sup _{\lambda \in[\gamma,+\infty}|\phi(\lambda)|$.

We denote by $S(y)=e^{-y \sqrt{A}}=\int_{\gamma}^{\infty} e^{-y \sqrt{\lambda}} d E_{\lambda} \in \mathscr{L}(H)$, $y \geq 0$, the $C_{0}$-semigroup generated by $-\sqrt{A}$. Some basic properties of $S(y)$ are listed in the following theorem.

Theorem 3 (see [17, chapter 2, Theorem 6.13, page 74]). For this family of operators one has:

(1) $\|S(y)\| \leq 1, \forall y \geq 0$;

(2) the function $y \mapsto S(y), y>0$, is analytic;

(3) for every real $r \geq 0$ and $y>0$, the operator $S(y) \in$ $\mathscr{L}\left(H, \mathscr{D}\left(A^{r / 2}\right)\right)$;

(4) for every integer $k \geq 0$ and $y>0,\left\|S^{(k)}(y)\right\|=$ $\left\|A^{k / 2} S(y)\right\| \leq c(k) y^{-k}$

(5) for every $x \in \mathscr{D}\left(A^{r / 2}\right), r \geq 0$, one has $S(t) A^{r / 2} x=$ $A^{r / 2} S(y) x$.

Theorem 4. For $y>0, S(y)$ is self-adjoint and one to one operator with dense range $\left(S(y)=S(y)^{*}, \overline{\mathscr{R}(S(y))}=H\right)$.

Proof. Let $\phi_{y}:\left[\gamma,+\infty\left[\rightarrow \mathbb{R}, s \mapsto \phi_{y}(s)=e^{-y s}\right.\right.$. Then by virtue of (iv) of Theorem 2 , we can write $S(y)^{*}=\overline{\phi_{y}}(A)=$ $\phi_{y}(A)=e^{-y \sqrt{A}}=S(y)$.

Let $h \in N\left(S\left(y_{0}\right)\right), y_{0}>0$, then $S\left(y_{0}\right) h=0$, which implies that $S(y) S\left(y_{0}\right) h=S\left(y+y_{0}\right) h=0, y \geq 0$. Using analyticity, we obtain that $S(y) h=0, y \geq 0$. Strong continuity at 0 now gives $h=0$. This shows that $N\left(S\left(y_{0}\right)\right)=\{0\}$.

Thanks to

$$
\overline{R\left(S\left(y_{0}\right)\right)}=N\left(S\left(y_{0}\right)\right)^{\perp}=\{0\}^{\perp}=H,
$$

we conclude that $R\left(S\left(y_{0}\right)\right)$ is dense in $H$.

Remark 5. For $y=p L, p \geq 1$, this Theorem ensures us that $S(p L)$ is self-adjoint and one to one operator with dense range $R(S(p L))$. Then we can define its inverse $S(p L)^{-1}=e^{p L \sqrt{A}}$, which is an unbounded self-adjoint strictly positive definite operator in $H$ with dense domain

$$
\begin{aligned}
D\left(S(p L)^{-1}\right) & =R(S(p L)) \\
& =\left\{h \in H:\left\|e^{p L \sqrt{A}} h\right\|^{2}\right. \\
& \left.=\int_{\gamma}^{+\infty} e^{2 p L \sqrt{\lambda}} d\left\|E_{\lambda} h\right\|^{2}<+\infty\right\} .
\end{aligned}
$$

Definition 6 (Hilbert scales). Let $B$ be an unbounded selfadjoint strictly positive definite operator in $H$. Following 
the definition in [18], one introduces the Hilbert scale $\left\{\mathscr{G}_{\theta}\right\}_{\theta \geq 0}$ according to definition

$$
\mathscr{G}_{0}=H, \quad \mathscr{G}_{\theta}=D\left(B^{\theta}\right) .
$$

Here $\mathscr{G}_{\theta}$ is a Hilbert space with inner product $(\xi, \zeta)_{\theta}=$ $\left(B^{\theta} \xi, B^{\theta} \zeta\right)$ and norm $\|\xi\|_{\theta}^{2}=\left(B^{\theta} \xi, B^{\theta} \xi\right)$. has

In our setting we take $B=S(L)^{-1}=e^{L \sqrt{A}}$. In this case, one

$$
\begin{aligned}
\mathscr{G}_{\theta}=\left\{h \in H:\|h\|_{\theta}^{2}\right. & =\left\|e^{\theta L \sqrt{A}} h\right\|^{2} \\
& \left.=\int_{\gamma}^{+\infty} e^{2 L \theta \sqrt{\lambda}} d\left\|E_{\lambda} h\right\|^{2}<+\infty\right\} .
\end{aligned}
$$

Following [15], one defines the following.

Definition 7 (mollification operator). For $\alpha>0$ and $p \geq 1$, one introduces the Yosida approximation of identity

$$
M_{\alpha}=\left(I+\alpha e^{p L \sqrt{A}}\right)^{-1}=\int_{\gamma}^{+\infty}\left(1+\alpha e^{p L \sqrt{\lambda}}\right)^{-1} d E_{\lambda} .
$$

Remark 8. The idea of the mollification method is very simple and natural: if the data are given inexactly, then we try to find a sequence of mollification operators which map the improper data into well-posed classes of the problem (mollify the improper data). Within these mollified data our problem becomes well-posed.

\section{Theorem 9. One has}

(1) $M_{\alpha} \in \mathscr{L}(H)$ and $\left\|M_{\alpha}\right\| \leq 1$,

(2) for all $h \in H, M_{\alpha} h \in \mathscr{G}_{p}$,

(3) for all $h \in H, \lim _{\alpha \rightarrow 0}\left\|M_{\alpha} h-h\right\|=0$.

Proof. (1) $\left\|M_{\alpha}\right\|=\sup _{\lambda \geq \gamma} 1 /\left(1+\alpha e^{p L \sqrt{\lambda}}\right)=1 /\left(1+\alpha e^{p L \sqrt{\gamma}}\right)<1$.

(2) $\left\|M_{\alpha} h\right\|_{p}^{2}=\int_{\gamma}^{+\infty}\left(e^{p L \sqrt{\lambda}} /\left(1+\alpha e^{p L \sqrt{\lambda}}\right)\right)^{2} d\left\|E_{\lambda} h\right\|^{2} \leq$ $(1 / \alpha)^{2}\|h\|^{2}<\infty \Leftrightarrow M_{\alpha} h \in \mathscr{G}_{p}$.

(3) Assume that $h \in \mathscr{G}_{p}$. Then

$$
\begin{aligned}
\left\|M_{\alpha} h-h\right\|^{2} & =\int_{\gamma}^{+\infty}\left(\frac{1}{1+\alpha e^{p L \sqrt{\lambda}}}-1\right)^{2} d\left\|E_{\lambda} h\right\|^{2} \\
& =\int_{\gamma}^{+\infty}\left(\frac{\alpha e^{p L \sqrt{\lambda}}}{1+\alpha e^{p L \sqrt{\lambda}}}\right)^{2} d\left\|E_{\lambda} h\right\|^{2} \leq \alpha^{2}\|h\|_{p}^{2},
\end{aligned}
$$

and thus, $\lim _{\alpha \rightarrow 0}\left\|M_{\alpha} h-h\right\|=0$.

Now for $h \in H$, there exists $h_{\varepsilon} \in \mathscr{G}_{p}$ such that $\left\|h-h_{\varepsilon}\right\| \leq$ $\varepsilon, \varepsilon>0$ (since $\mathscr{G}_{p}$ is dense in $H$ (see Remark 5)). We have

$$
\begin{aligned}
& \left\|M_{\alpha} h-h\right\| \\
& \quad=\left\|M_{\alpha} h-M_{\alpha} h_{\varepsilon}+M_{\alpha} h_{\varepsilon}-h_{\varepsilon}+h_{\varepsilon}-h\right\| \\
& \quad \leq\left\|M_{\alpha}\left(h-h_{\varepsilon}\right)\right\|+\left\|M_{\alpha} h_{\varepsilon}-h_{\varepsilon}\right\|+\left\|h_{\varepsilon}-h\right\| \\
& \quad \leq 2 \varepsilon+\left\|M_{\alpha} h_{\varepsilon}-h_{\varepsilon}\right\| .
\end{aligned}
$$

Thus, $\lim _{\alpha \rightarrow 0}\left\|M_{\alpha} h-h\right\| \leq 2 \varepsilon, \forall \varepsilon>0$, and so $\lim _{\alpha \rightarrow 0} \| M_{\alpha} h-$ $h \|=0$.

Using the change of variables

$$
\begin{aligned}
U(y) & =\frac{1}{2}\left(u(y)-A^{-1 / 2} u^{\prime}(y)\right), \\
W(y) & =\frac{1}{2}\left(u(y)+A^{-1 / 2} u^{\prime}(y)\right) .
\end{aligned}
$$

Cauchy's problem (1) reduces to the two Cauchy problems

$$
\begin{gathered}
U^{\prime}(y)=-A^{1 / 2} U(y), \quad 0<y<L, \\
U(0)=\frac{1}{2}\left(u(0)-A^{-1 / 2} u^{\prime}(0)\right)=\frac{1}{2} f, \\
W^{\prime}(y)=A^{1 / 2} W(y), \quad 0<y<L, \\
W(0)=\frac{1}{2}\left(u(0)+A^{-1 / 2} u^{\prime}(0)\right)=\frac{1}{2} f .
\end{gathered}
$$

Thus, the solution of the original problem (1) can be written in the form

$$
u(y)=U(y)+W(y), \quad 0 \leq y \leq L
$$

It is well known that the operator $-A^{1 / 2}$ generates a strongly continuous analytic semigroup $S_{-1 / 2}(y)=\int_{\gamma}^{+\infty} e^{-y \sqrt{\lambda}} d E_{\lambda}$. In addition, the spectral radius of the semigroup $\left\|S_{-1 / 2}(y)\right\|<1$ for any $y>0$. Hence, it follows that the Cauchy problem (15) is well-posed and its solution may be written in the form

$$
\begin{aligned}
U(y) & =S_{-1 / 2}(y) U(0) \\
& =\frac{1}{2} e^{-y \sqrt{A}} f=\frac{1}{2} \int_{\gamma}^{+\infty} e^{-y \sqrt{\lambda}} d E_{\lambda} f .
\end{aligned}
$$

As opposed to problem (15), Cauchy problem (15) (backward parabolic equation) is not correctly posed, and its (unique) formal solution is given by

$$
\begin{aligned}
W(y) & =S_{+1 / 2}(y) W(0) \\
& =\frac{1}{2} e^{y \sqrt{A}} f=\frac{1}{2} \int_{\gamma}^{+\infty} e^{y \sqrt{\lambda}} d E_{\lambda} f .
\end{aligned}
$$

Remark 10 (see [19, page 375$]$ ). The uniqueness solvability of problem (16) results from the logarithmic convexity of the function $y \mapsto\|W(y)\|$ :

$$
\forall y \in[0, L], \quad\|W(y)\| \leq\|W(0)\|^{1-y / L}\|W(L)\|^{y / L} .
$$

A useful characterization of the admissible set for which problem (16) has a solution is as follows.

Lemma 11. Problem (16) has a solution $W \in C([0, L] ; H)$ if and only if $f \in \mathscr{G}_{1}$, and its unique solution is represented by

$$
W(y)=S_{+1 / 2}(y) W(0)=\frac{1}{2} e^{y \sqrt{A}} f=\frac{1}{2} \int_{\gamma}^{+\infty} e^{y \sqrt{\lambda}} d E_{\lambda} f .
$$


Proof. Suppose that problem (16) has a solution $W \in$ $\mathscr{C}([0, L] ; H)$. Then

$$
W(y)=\frac{1}{2} \int_{\gamma}^{+\infty} e^{y \sqrt{\lambda}} d E_{\lambda} f
$$

The function $W \in \mathscr{C}([0, L] ; H)$ if and only if

$$
\sup _{y \in[0, L]}\|W(y)\|^{2}=\frac{1}{4} \int_{\gamma}^{+\infty} e^{2 y \sqrt{\lambda}} d\left\|E_{\lambda} f\right\|^{2}<\infty .
$$

Observing that the function $y \mapsto\|W(L)\|^{2}$ is increasing, then

$$
\sup _{y \in[0, L]}\|W(y)\|^{2}=\|W(L)\|^{2}=\frac{1}{4} \int_{\gamma}^{+\infty} e^{2 L \sqrt{\lambda}} d\left\|E_{\lambda} f\right\|^{2}<\infty .
$$

This last inequality is exactly equivalent to $f \in \mathscr{G}_{1}$.

As a consequence, we have the following corollary.

Corollary 12. Problem (1) has a solution $u \in C([0, L] ; H)$ if and only if $f \in \mathscr{G}_{1}$, and its unique solution is represented by

$$
u(y)=U(y)+W(y)=\frac{1}{2} \int_{\gamma}^{+\infty}\left(e^{y \sqrt{\lambda}}+e^{-y \sqrt{\lambda}}\right) d E_{\lambda} f .
$$

\section{Regularization and Error Estimates}

3.1. The Truncation Method. From (25) we can see that the term $e^{y \sqrt{\lambda}}$ is the cause of unstability. In order to overcome the ill-posedness of problem (1), we modify the solution by filtering the high frequencies using a suitable method and instead consider (25) only for $\lambda \leq \beta(\delta)$, where $\beta(\delta)$ is some constant which satisfies $\lim _{\delta \rightarrow 0} \beta(\delta)=+\infty$.

According to spectral theory of self-adjoint operators [20], for any bounded Borel set $\Delta_{\beta}=\{\gamma \leq t \leq \beta\} \subseteq \sigma(A)=$ $[\gamma,+\infty[$, we can define the orthogonal projection

$$
\mathbf{1}_{\Delta_{\beta}}=\int_{\gamma}^{+\infty} \mathbf{1}_{\Delta_{\beta}}(\lambda) d E_{\lambda}=E_{\beta}
$$

$\forall h \in H, \quad h_{\beta}=E_{\beta} h \longrightarrow h, \quad \beta \longrightarrow+\infty$.

To solve (1) in a stable way we approximate $f$ by its projection $f_{\beta}$, and instead of considering (1) with $f$ we take its projected version

$$
\begin{aligned}
u_{\beta}(y) & =\cosh (y \sqrt{A}) f_{\beta} \\
& =\frac{1}{2} \int_{\gamma}^{+\infty}\left(e^{y \sqrt{\lambda}}+e^{-y \sqrt{\lambda}}\right) \mathbf{1}_{[\gamma, \beta]} d E_{\lambda} f
\end{aligned}
$$

where $\mathbf{1}_{[a, b]}$ is the characteristic function of the interval $[a, b]$ for $a<b$. The quantity $\beta$ is referred to as a cut-off frequency.

Let $f$ (resp., $f_{\delta}$ ) be the exact (resp., the measured data) at $y=0$, such that $\left\|f-f_{\delta}\right\| \leq \delta$.
The approximated solution $v_{\beta}^{\delta}$ corresponding to the measured data $f_{\delta}$ is denoted by

$$
v_{\beta}^{\delta}(y)=\frac{1}{2} \int_{\gamma}^{+\infty}\left(e^{y \sqrt{\lambda}}+e^{-y \sqrt{\lambda}}\right) \mathbf{1}_{[\gamma, \beta]} d E_{\lambda} f_{\delta} .
$$

For simplicity, we denote the solution of problem (1) by $u(y)$, and the regularized solution associated to the data $f_{\delta}$ by $v_{\beta}^{\delta}(y)$.

Our first main theorem is the following theorem.

Theorem 13. The solution defined in (27) depends continuously in $\mathscr{C}([0, L] ; H)$ on the data $f$; that is, if $u_{\beta}^{1}$ and $u_{\beta}^{2}$ are two regularized solutions corresponding to $f_{1}$ and $f_{2}$, respectively, then one has

$$
\left\|u_{\beta}^{1}(y)-u_{\beta}^{2}(y)\right\| \leq e^{y \sqrt{\beta}}\left\|f_{1}-f_{2}\right\| .
$$

This inequality implies that the solution of the regularized problem (27) depends continuously on the data $f$.

Now we compute the difference between the original solution $u=u(y ; f)$ and the approximate solution $v_{\beta}^{\delta}=$ $v_{\beta}^{\delta}\left(y ; f_{\delta}\right)$.

Theorem 14. Let $u \in C([0, L] ; H)$ be a solution of problem $(1)$ with the exact data $f \in H$; then the following estimate holds:

$$
\left\|u(y)-u_{\beta}(y)\right\| \leq \frac{2}{e^{(L-y) \sqrt{\beta}}}\|u(L)\| .
$$

Proof. From relations (25) and (27) we have

$$
\begin{aligned}
u(y)-u_{\beta}(t) & =\int_{\beta}^{+\infty} \cosh (y \sqrt{\lambda}) d E_{\lambda} f \\
& =\int_{\gamma}^{+\infty} \cosh (y \sqrt{\lambda}) \mathbf{1}_{[\beta,+\infty]} d E_{\lambda} f
\end{aligned}
$$

then

$$
\begin{aligned}
& u(y)-u_{\beta}(y) \\
& =\int_{\gamma}^{+\infty} \frac{\cosh (y \sqrt{\lambda})}{\cosh (L \sqrt{\lambda})} \mathbf{1}_{[\beta,+\infty]} \cosh (L \sqrt{\lambda}) d E_{\lambda} f, \\
& \left\|u(y)-u_{\beta}(y)\right\|^{2} \\
& \quad \leq \int_{\gamma}^{+\infty}\left(\frac{\cosh (y \sqrt{\lambda})}{\cosh (L \sqrt{\lambda})} \mathbf{1}_{[\beta,+\infty]}\right)^{2} \cosh (L \sqrt{\lambda})^{2} d\left\|E_{\lambda} f\right\|^{2} .
\end{aligned}
$$

Using the inequality

$$
\begin{aligned}
& \left(\frac{\cosh (y \sqrt{\lambda})}{\cosh (L \sqrt{\lambda})} \mathbf{1}_{[\beta,+\infty]}\right)^{2} \leq \frac{4}{e^{2(L-y) \sqrt{\beta}}}, \\
& \int_{\beta}^{+\infty} \cosh ^{2}(L \sqrt{\lambda}) d\left\|E_{\lambda} f\right\|^{2} \leq\|u(L)\|^{2},
\end{aligned}
$$


we derive

$$
\left\|u(y)-u_{\beta}(y)\right\|^{2} \leq \frac{4}{e^{2(L-y) \sqrt{\beta}}}\|u(L)\|^{2} .
$$

Using (29), (30) and the triangle inequality, we obtain

$$
\begin{aligned}
& \left\|u(y)-v_{\beta}^{\delta}(y)\right\| \\
& \quad \leq\left\|u(y)-u_{\beta}(y)\right\|+\left\|u_{\beta}(y)-v_{\beta}^{\delta}(y)\right\| \\
& \quad \leq \frac{2}{e^{(L-y) \sqrt{\beta}}}\|u(L)\|+e^{y \sqrt{\beta}} \delta .
\end{aligned}
$$

This completes the proof.

Remark 15. If we choose $\sqrt{\beta}=(1 / L) \log (M / \delta)$, where $\|u(L)\|=M$, then we have the error bound

$$
\left\|u(y)-v_{\beta}^{\delta}(y)\right\| \leq 3 M^{(L-y) / L} \delta^{y / L} .
$$

From (36) we see that (28) is an approximation of the exact solution $u(y)$. The approximation error depends continuously on the measurement error for fixed $0<y<L$. However, as $y \rightarrow L$, the accuracy of the regularized solution becomes progressively lower. Consequently, we have not any information about the continuous dependence of the solution if $y$ is close to $L$.

In the theory of ill-posed Cauchy problems, we can often obtain continuous dependence on the data for the closed interval $[0, L]$ by assuming additional smoothness and using a stronger norm.

Now we show two error estimates under the following conditions:

$$
\begin{aligned}
& \text { (H1) } u(L) \in D\left(A^{p}\right), \\
& (\mathrm{H} 2) u(L) \in \mathscr{G}_{p}, p>0 .
\end{aligned}
$$

Remark 16. In practice, we know that it is very difficult to verify the conditions ( $\mathrm{H} 1)$ and $(\mathrm{H} 2)$, so we give different assumptions on the given data $f$ as follows:

$$
\begin{aligned}
u(L) \in D\left(A^{p}\right) & \Longleftrightarrow \int_{\gamma}^{+\infty} \lambda^{2 p} \cosh ^{2}(L \sqrt{\lambda}) d\left\|E_{\lambda} f\right\|^{2}<\infty \\
& \Longleftrightarrow \int_{\gamma}^{+\infty} \lambda^{2 p} e^{2 L \sqrt{\lambda}} d\left\|E_{\lambda} f\right\|^{2}<\infty, \\
u(L) \in \mathscr{G}_{p} & \Longleftrightarrow \int_{\gamma}^{+\infty} e^{2 p L \sqrt{\lambda}} \cosh ^{2}(L \sqrt{\lambda}) d\left\|E_{\lambda} f\right\|^{2}<\infty \\
& \Longleftrightarrow \int_{\gamma}^{+\infty} e^{2(1+p) L \sqrt{\lambda}} d\left\|E_{\lambda} f\right\|^{2}<\infty \\
& \Longleftrightarrow f \in \mathscr{G}_{p+1} .
\end{aligned}
$$

Theorem 17. If $\int_{\gamma}^{+\infty} \lambda^{p} e^{2 L \sqrt{\lambda}} d\left\|E_{\lambda} f\right\|^{2} \quad<\quad E_{1}^{2}$ (resp., $\left.\int_{\gamma}^{+\infty} e^{2(1+q) L \sqrt{\lambda}} d\left\|E_{\lambda} f\right\|^{2}<E_{2}^{2}\right), p>0, q>0$, then one has the following estimates:

$$
\begin{gathered}
\left\|u(y)-v_{\beta}^{\delta}(y)\right\| \\
\leq\left(\frac{L}{a}\right)^{p} E_{1} \log \left(\frac{1}{\delta}\right)^{-p}+\delta^{1-y a / L}, \quad 0<a \leq 1, \\
\left\|u(y)-v_{\beta}(y)\right\| \leq e^{-q \sqrt{\beta}} E_{2}+e^{y \sqrt{\beta}} \delta .
\end{gathered}
$$

Proof. From the expansions

$$
\begin{gathered}
u(y)=\int_{\gamma}^{\infty} \cosh (y \sqrt{\lambda}) d E_{\lambda} f, \\
u_{\beta}(y)=\int_{\gamma}^{\infty} \cosh (y \sqrt{\lambda}) \mathbf{1}_{[\gamma, \beta]} d E_{\lambda} f
\end{gathered}
$$

we have

$$
u(y)-u_{\beta}(y)=\int_{\gamma}^{\infty} \cosh (y \sqrt{\lambda}) \mathbf{1}_{[\beta,+\infty]} d E_{\lambda} f .
$$

Then

$$
\begin{aligned}
& \left\|u(y)-u_{\beta}(y)\right\|^{2} \\
& =\int_{\gamma}^{\infty}\left(\lambda^{-p / 2} \mathbf{1}_{[\beta,+\infty]}\right)^{2} \cosh ^{2}(y \sqrt{\lambda}) \lambda^{p} d\left\|E_{\lambda} f\right\|^{2} \\
& \leq \int_{\gamma}^{\infty}\left(\lambda^{-p / 2} \mathbf{1}_{[\beta,+\infty]}\right)^{2} e^{2 L \sqrt{\lambda}} \lambda^{p} d\left\|E_{\lambda} f\right\|^{2} \\
& \leq \beta^{-p} \int_{\gamma}^{\infty} e^{2 L \sqrt{\lambda}} \lambda^{p} d\left\|E_{\lambda} f\right\|^{2} \\
& \leq \sqrt{\beta}^{-2 p} E_{1}^{2} .
\end{aligned}
$$

Using Theorem 17 and the triangle inequality, we can write

$$
\begin{aligned}
& \left\|u(y)-v_{\beta}^{\delta}(y)\right\| \\
& \quad \leq\left\|u(y)-u_{\beta}(y)\right\|+\left\|u_{\beta}(y)-v_{\beta}^{\delta}(y)\right\| \\
& \quad \leq \sqrt{\beta}^{-p} E_{1}+e^{y \sqrt{\beta}} \delta .
\end{aligned}
$$

By choosing $\sqrt{\beta}=(a / L) \log (1 / \delta)$, we obtain the desired inequality.

Using the same techniques we have

$$
\begin{aligned}
& u(y)-u_{\beta}(y) \\
& \quad=\int_{\gamma}^{\infty} e^{-q \sqrt{\lambda}} \cosh (y \sqrt{\lambda}) e^{q \sqrt{\lambda}} \mathbf{1}_{[\beta,+\infty]} d E_{\lambda} f,
\end{aligned}
$$


hence

$$
\begin{aligned}
& \left\|u(y)-u_{\beta}(y)\right\|^{2} \\
& =\int_{\gamma}^{\infty}\left(e^{-q \sqrt{\lambda}} \mathbf{1}_{[\beta,+\infty]}\right)^{2}\left(\cosh (y \sqrt{\lambda}) e^{2 q \sqrt{\lambda}}\right)^{2} d\left\|E_{\lambda} f\right\|^{2} \\
& \leq e^{-2 q \sqrt{\beta}} \int_{\gamma}^{\infty} e^{2 q \sqrt{\lambda}} d\left\|E_{\lambda} u(y)\right\|^{2} \leq e^{-2 q \sqrt{\beta}} E_{2}^{2} .
\end{aligned}
$$

Using (29) and the triangle inequality, we obtain

$$
\begin{aligned}
& \left\|u(y)-v_{\beta}^{\delta}(y)\right\| \\
& \quad \leq\left\|u(y)-u_{\beta}(y)\right\|+\left\|u_{\beta}(y)-v_{\beta}^{\delta}(y)\right\| \\
& \quad \leq e^{-q \sqrt{\beta}} E_{2}+e^{y \sqrt{\beta}} \delta .
\end{aligned}
$$

By choosing $\sqrt{\beta}=(a / L) \log (1 / \delta)$, we obtain

$$
\left\|u(y)-v_{\beta}^{\delta}(y)\right\| \leq \delta^{a q / L} E_{2}+\delta^{1-a y / L} .
$$

3.2. The Mollification Method. Now, we approximate the original problem (1) by the sequence of problems

$$
\begin{gathered}
u_{y y}=A u, \quad 0<y<L, \\
u(0)=f_{\alpha}=M_{\alpha} f, \\
u_{y}(0)=0 .
\end{gathered}
$$

Theorem 18. If $f \in H$ the approximate Cauchy problem (47) admits a unique solution $u_{\alpha}$, which depends continuously upon the data $f$ with respect to uniform topology of $C([0, L] ; H)$.

Proof. From the representation

$$
\begin{aligned}
u_{\alpha}(y) & =\cosh (y \sqrt{A}) f_{\alpha} \\
& =\int_{\gamma}^{+\infty} \cosh (y \sqrt{\lambda})\left(1+\alpha e^{p L \sqrt{\lambda}}\right)^{-1} d E_{\lambda} f
\end{aligned}
$$

we have

$$
\begin{aligned}
\left\|u_{\alpha}(y)\right\|^{2} & =\int_{\gamma}^{+\infty}\left\{\frac{\cosh (y \sqrt{\lambda})}{1+\alpha e^{p L \sqrt{\lambda}}}\right\}^{2} d\left\|E_{\lambda} f\right\|^{2} \\
& \leq \int_{\gamma}^{+\infty}\left\{\frac{e^{L \sqrt{\lambda}}}{1+\alpha e^{p L \sqrt{\lambda}}}\right\}^{2} d\left\|E_{\lambda} f\right\|^{2} .
\end{aligned}
$$

(i) If $p=1$, we obtain

$$
\sup _{y \in[0, L]}\left\|u_{\alpha}(y)\right\|^{2} \leq \frac{1}{\alpha}\|f\| .
$$

(ii) If $p>1$, the function $M(s)=e^{L s} /\left(1+\alpha e^{p L s}\right)$ with $s=$ $\sqrt{\lambda} \geq \sqrt{\gamma}$ achieves its maximum at $s^{*}=(1 / p L) \log (1 /$ $\alpha(p-1)), p>1$, from which we deduce

$$
\begin{gathered}
M_{\infty}=M\left(s^{*}\right)=c(p)\left(\frac{1}{\alpha}\right)^{1 / p}, \\
c(p)=p^{-1}(p-1)^{1-1 / p} \leq 1 .
\end{gathered}
$$

From this bound, we derive

$$
\sup _{y \in[0, L]}\left\|u_{\alpha}(y)\right\| \leq\left(\frac{1}{\alpha}\right)^{1 / p}\|f\| .
$$

From the linear property of our problem, stability estimate of problem (47) may be written precisely in the following corollary.

Corollary 19. If $u_{\alpha, 1}\left(y ; f_{1}\right)\left(\right.$ resp., $\left.u_{\alpha, 2}\left(y ; f_{2}\right)\right)$ is the approximate solution corresponding to $f_{1}$ (resp., $f_{2}$ ), then

$$
\sup _{y \in[0, L]}\left\|u_{\alpha, 1}(y)-u_{\alpha, 2}(y)\right\| \leq\left(\frac{1}{\alpha}\right)^{1 / p}\left\|f_{1}-f_{2}\right\| .
$$

Remark 20. We have

$$
\begin{gathered}
N(s)=\frac{s^{r} e^{T s}}{1+\alpha e^{p T s}} \leq \frac{1}{\alpha} K(s) \\
=\frac{1}{\alpha} s^{r} e^{-(p-1) T s}, \quad p>1, \\
s=\sqrt{\lambda} \geq \sqrt{\gamma} .
\end{gathered}
$$

It is easy to show that

$$
\begin{aligned}
K(s) & \leq K\left(s^{*}=\frac{r}{L(p-1)}\right) \\
& =\left(\frac{r}{L(p-1)}\right)^{r} e^{-r}=\kappa(r, p, L)<\infty .
\end{aligned}
$$

This remark shows that $u_{\alpha}(y) \in D\left(A^{r / 2}\right)$ for all $y \in[0, L]$.

Proof. The inclusion $u_{\alpha}(y) \in D\left(A^{r / 2}\right)$ is equivalent to $\left\|A^{r / 2} u_{\alpha}(y)\right\|<\infty$. We have

$$
\begin{aligned}
\left\|A^{r / 2} u_{\alpha}(y)\right\|^{2} & =\int_{\gamma}^{+\infty} \lambda^{r}\left\{\frac{\cosh (y \sqrt{\lambda})}{1+\alpha e^{p L \sqrt{\lambda}}}\right\}^{2} d\left\|E_{\lambda} f\right\|^{2} \\
& \leq\left(\frac{1}{\alpha}\right)^{2} \int_{\gamma}^{+\infty}\left\{\sqrt{\lambda}^{r} e^{-(p-1) L \sqrt{\lambda}}\right\}^{2} d\left\|E_{\lambda} f\right\|^{2} \\
& \leq\left(\frac{1}{\alpha}\right)^{2} \kappa(r, p, L)^{2}\|f\|^{2}<\infty,
\end{aligned}
$$

where $\kappa(r, p, L)=\sup _{\lambda \geq \gamma} \sqrt{\lambda}^{r} e^{-(p-1) L \sqrt{\lambda}}=\left(\frac{r}{(p-1) L}\right)^{r} e^{-r}$. 
Theorem 21. If $f \in \mathscr{G}_{1}$, then

$$
\sup _{y \in[0, L]}\left\|u(y)-u_{\alpha}(y)\right\| \longrightarrow 0, \quad \alpha \longrightarrow 0 .
$$

Proof. We compute

$$
\begin{aligned}
& \left\|u(y)-u_{\alpha}(y)\right\|^{2} \\
& \quad=\int_{\gamma}^{+\infty}\left(1-M_{\alpha}(\lambda)\right)^{2} \cosh ^{2}(y \sqrt{\lambda}) d\left\|E_{\lambda} f\right\|^{2} \\
& \quad \leq \int_{\gamma}^{+\infty}\left(1-M_{\alpha}(\lambda)\right)^{2} \cosh ^{2}(L \sqrt{\lambda}) d\left\|E_{\lambda} f\right\|^{2} \\
& \leq \int_{\gamma}^{+\infty}\left(1-M_{\alpha}(\lambda)\right)^{2} e^{2 L \sqrt{\lambda}} d\left\|E_{\lambda} f\right\|^{2} \\
& \quad=\left\|\left(I-M_{\alpha}\right) \hat{f}\right\|^{2},
\end{aligned}
$$

where $\widehat{f}=e^{L \sqrt{A}} f$ and $\|\widehat{f}\|^{2}=\int_{\gamma}^{+\infty} e^{2 L \sqrt{\lambda}} d\left\|E_{\lambda} f\right\|^{2}<\infty$.

This implies that $\sup _{y \in[0, L]}\left\|u(y)-u_{\alpha}(y)\right\| \leq\left\|\left(I-M_{\alpha}\right) \widehat{f}\right\|$ and by virtue of (3) of Theorem 9 , we conclude the desired convergence.

The following technical lemmas play the key role in our analysis and calculations.

Lemma 22. Let

$$
\left[v,+\infty\left[\ni s \longmapsto Q(\{a, r, q, L\} ; s)=\frac{1}{\alpha s^{r}+a e^{-q L s}},\right.\right.
$$

where $a>0, \alpha>0, v>0, q>0, L>0$, and $r \geq 1$. Then one has

$$
Q(\{a, r, q, L\} ; s) \leq \frac{1}{\alpha}\left(\frac{k_{1}}{\log \left(k_{2}(1 / \alpha)\right)}\right)^{r}
$$

where $k_{1}(r, q, L)=r q L, k_{2}(q, r, L, a)=q^{r} L^{r-1} a / r$.

Proof. Differentiating the expression and setting the derivative equal to zero, we find

$$
\begin{aligned}
& \frac{d}{d s} Q(\{a, r, q, L\} ; s) \\
& \quad=\frac{-1}{\left(\alpha s^{r}+a e^{-q L s}\right)^{2}}\left(\alpha r s^{r-1}-q a e^{-q L s}\right)=0 .
\end{aligned}
$$

The function $(d / d s) Q(\{a, r, q, L\} ; s)=0$ admits a unique solution

$$
\widehat{s}=\left\{s \longmapsto \alpha r s^{r-1}\right\} \cap\left\{s \longmapsto q a e^{-q L s}\right\}
$$

Therefore

$$
\begin{aligned}
Q(\{a, r, q, L\} ; s) & \leq Q(\{a, r, q, L\} ; \widehat{s}) \\
& \leq \frac{1}{\alpha \widehat{s}^{r}+a e^{-q L \widehat{s}}} \leq \frac{1}{\alpha \widehat{s}^{r}} .
\end{aligned}
$$

We have

$$
\left(\alpha r \widehat{s}^{r-1}-q a e^{-q L \widehat{s}}\right)=0 \Longleftrightarrow s^{r-1} e^{q L \widehat{s}}=\frac{q a}{r \alpha} .
$$

By using the inequality ( $\left.e^{t} \geq t, t \geq 0\right)$, then for $t=q L \widehat{s}$, we obtain $e^{q L \widehat{s}} \geq q L \widehat{s}$ and we can write

$$
\frac{q a}{r \alpha}=\widehat{s}^{r-1} e^{q L \widehat{s}} \leq e^{q L \hat{s}}\left(\frac{e^{q L \widehat{s}}}{q L}\right)^{r-1}=\left(\frac{1}{q L}\right)^{r-1} e^{r q L \widehat{s}},
$$

which implies that $\widehat{s} \geq(1 / r q L) \log \left(\left(q^{r L^{r-1} a} / r\right)(1 / \alpha)\right)$. Hence, we obtain

$$
Q(\{a, r, q, L\} ; s) \leq \frac{1}{\alpha \widehat{s}^{r}} \leq \frac{1}{\alpha}\left(\frac{k_{1}}{\log \left(k_{2}(1 / \alpha)\right)}\right)^{r},
$$

where $k_{1}(r, q, L)=r q L, k_{2}(q, r, L, a)=q^{r} L^{r-1} a / r$.

Lemma 23. Let

$$
\begin{aligned}
{[\nu,+\infty[\ni s \longmapsto R(\{p, q, L\} ; s)} \\
\quad=\frac{e^{p L s}}{\left(1+\alpha e^{p L s}\right) e^{q L s}}=\frac{1}{e^{(q-p) L s}+\alpha e^{q L s}},
\end{aligned}
$$

where $p \geq 1, q>0, \alpha>0, v>0$, and $L>0$. Then one has the following.

If $1 \leq p \leq q$, then

$$
R(\{p, q, L\} ; s) \leq e^{-(q-p) L s} \leq e^{-(q-p) L v} \leq 1 .
$$

If $0<q<p, p \geq 1,0<\alpha \leq(p-q) / q$, then

$$
\begin{gathered}
R(\{p, q, L\} ; s) \leq k_{3}\left(\frac{1}{\alpha}\right)^{(p-q) / p}, \\
k_{3}(p, q)=\frac{q}{p}\left(\frac{p-q}{p}\right)^{(p-q) / p} \leq 1 .
\end{gathered}
$$

Proof. By a simple differential calculus, we show that the function $R(\{p, q, L\} ; s)$ achieves its maximum at $\widehat{s}=(1 /$ $p L) \log ((p-q) / \alpha q)$. Consequently

$$
R(\{p, q, L\} ; s) \leq R(\{p, q, L\} ; \widehat{s})=k_{3}\left(\frac{1}{\alpha}\right)^{(p-q) / p} .
$$

Now we assume the following a priori bounds hold:

$$
\begin{aligned}
u(L) & \in D\left(A^{r / 2}\right) \\
& \Longleftrightarrow \int_{\gamma}^{+\infty} \sqrt{\lambda}^{2 r} e^{2 L \sqrt{\lambda}} d\left\|E_{\lambda} f\right\|^{2} \leq E_{1}^{2}<\infty, \\
u(L) \in \mathscr{G}_{q} & \Longleftrightarrow \int_{\gamma}^{+\infty} e^{2 L(1+q) \sqrt{\lambda}} d\left\|E_{\lambda} f\right\|^{2} \leq E_{2}^{2}<\infty .
\end{aligned}
$$


Theorem 24. Let $u$ (resp., $u_{\alpha}$ ) be the solution of problem (1) (resp., (47)) with the exact data $f$. If (71) (resp., (72)) is satisfied, then one has the following error estimates:

$$
\begin{gathered}
\left\|u(y)-u_{\alpha}(y)\right\|=O\left(\frac{1}{\log (1 / \alpha)}\right)^{r}, \\
\left\|u(y)-u_{\alpha}(y)\right\| \\
= \begin{cases}O(\alpha), & \text { if } 1 \leq p \leq q, \\
O\left(\alpha^{q / p}\right), & \text { if } 0<q<p, p \geq 1 .\end{cases}
\end{gathered}
$$

Proof. Putting

$$
\begin{aligned}
& B_{1}(\lambda)=\left\{\frac{e^{p L \sqrt{\lambda}}}{1+\alpha e^{p L \sqrt{\lambda}}}\right\} \frac{1}{\sqrt{\lambda}^{r}} \\
&=\frac{1}{\sqrt{\lambda}^{r} e^{-p L \sqrt{\lambda}}+\alpha \sqrt{\lambda}^{r}} \leq B_{2}(\lambda) \\
&=\frac{1}{\sqrt{\gamma}^{r} e^{-p L \sqrt{\lambda}}+\alpha \sqrt{\lambda}^{r}}, \\
& B_{3}(\lambda)=\left\{\frac{e^{p L \sqrt{\lambda}}}{1+\alpha e^{p L \sqrt{\lambda}}}\right\} \frac{1}{e^{q L \sqrt{\lambda}}}=\frac{1}{e^{(q-p) L \sqrt{\lambda}}+\alpha e^{q L \sqrt{\lambda}}} .
\end{aligned}
$$

Using the change of variables $s=\sqrt{\lambda}$, we obtain the new expressions

$$
\begin{aligned}
& \widehat{B}_{2}(s)=\frac{1}{\sqrt{\gamma}^{r} e^{-p L s}+\alpha s^{r}}, \\
& \widehat{B}_{3}(s)=\frac{1}{e^{(q-p) L s}+\alpha e^{q L s}} .
\end{aligned}
$$

By virtue of Lemma 22 (inequality (60) and Lemma 23 (inequalities (68) and (69)), we can write

$$
\widehat{B}_{2}(s) \leq \frac{1}{\alpha}\left(\frac{k_{1}}{\log \left(k_{2}(1 / \alpha)\right)}\right)^{r}
$$

where $k_{1}(r, p, L)=r q L, k_{2}\left(p, r, L, \sqrt{\gamma}^{r}\right)=q^{r} L^{r-1} \sqrt{\gamma}^{r} / r$. Consider

$$
\widehat{B}_{3}(s) \leq \begin{cases}1, & \text { if } 1 \leq p \leq q, \\ \left(\frac{1}{\alpha}\right)^{(p-q) / p}, & \text { if } 0<q<p, p \geq 1 .\end{cases}
$$

We have

$$
\begin{aligned}
& \left\|u(y)-u_{\alpha}(y)\right\|^{2} \\
& =\int_{\gamma}^{+\infty}\left\{\frac{\alpha e^{p L \sqrt{\lambda}}}{1+\alpha e^{p L \sqrt{\lambda}}}\right\}^{2} \cosh ^{2}(y \sqrt{\lambda}) d\left\|E_{\lambda} f\right\|^{2} \\
& \leq \alpha^{2} \int_{\gamma}^{+\infty}\left\{B_{2}(\lambda)\right\}^{2} \sqrt{\lambda}^{2 r} e^{2 L \sqrt{\lambda}} d\left\|E_{\lambda} f\right\|^{2} \\
& \leq \alpha^{2}\left(\sup _{s \geq \sqrt{\gamma}} \widehat{B}_{2}(s)\right)^{2} E_{1}^{2}, \\
& \left\|u(y)-u_{\alpha}(y)\right\|^{2} \\
& \quad=\int_{\gamma}^{+\infty}\left\{\frac{\alpha e^{p L \sqrt{\lambda}}}{1+\alpha e^{p L \sqrt{\lambda}}}\right\}^{2} \cosh ^{2}(y \sqrt{\lambda}) d\left\|E_{\lambda} f\right\|^{2} \\
& \leq \alpha^{2} \int_{\gamma}^{+\infty}\left\{B_{3}(\lambda)\right\}^{2} e^{L(1+q) \sqrt{\lambda}} d\left\|E_{\lambda} f\right\|^{2} \\
& \quad \leq \alpha^{2}\left(\sup _{s \geq \sqrt{\gamma}} \widehat{B}_{3}(s)\right)^{2} E_{2}^{2} .
\end{aligned}
$$

Using (78) and (79), we derive

$$
\begin{gathered}
\left\|u(y)-u_{\alpha}(y)\right\| \leq \alpha \frac{1}{\alpha}\left(\frac{k_{1}}{\log \left(k_{2}(1 / \alpha)\right)}\right)^{r}=O\left(\frac{1}{\log (1 / \alpha)}\right)^{r}, \\
\left\|u(y)-u_{\alpha}(y)\right\| \leq \begin{cases}\alpha, & \text { if } 1 \leq p \leq q, \\
\alpha^{q / p}, & \text { if } 0<q<p, p \geq 1 .\end{cases}
\end{gathered}
$$

Combining (53), (73), and (74) with the help of triangle inequality

$$
\begin{aligned}
&\left\|u(y)-u_{\alpha}^{\delta}(y)\right\| \\
& \quad \leq\left\|u(y)-u_{\alpha}(y)\right\| \\
&+\left\|u_{\alpha}(y)-u_{\alpha}^{\delta}(y)\right\|=\Delta_{1}+\Delta_{2},
\end{aligned}
$$

we deduce the following corollary.

Corollary 25. Let $u(y ; f)\left(\right.$ resp., $\left.u_{\alpha}^{\delta}\left(y ; f_{\delta}\right)\right)$ be the solution of problem (1) (resp., (47)) with the exact data $f$ (resp., the inexact data $f_{\delta}$ ) such that $\left\|f-f_{\delta}\right\| \leq \delta$. If (71) (resp., (72)) is satisfied, then one has the following error estimates:

(case $r \geq 1$ )

$$
\left\|u(y)-u_{\alpha}^{\delta}(y)\right\|=O\left(\theta_{1}(\alpha)\right)+\left(\frac{1}{\alpha}\right)^{1 / p} \delta,
$$




$$
\begin{aligned}
& (\text { case } 1 \leq p \leq q) \\
& \left\|u(y)-u_{\alpha}^{\delta}(y)\right\|=O\left(\theta_{2}(\alpha)\right)+\left(\frac{1}{\alpha}\right)^{1 / p} \delta, \\
& (\text { case } 0<q<p, p \geq 1) \\
& \left\|u(y)-u_{\alpha}^{\delta}(y)\right\|=O\left(\theta_{3}(\alpha)\right)+\left(\frac{1}{\alpha}\right)^{1 / p} \delta,
\end{aligned}
$$

where

$$
\begin{gathered}
\theta_{1}(\alpha)=O\left(\frac{1}{\log (1 / \alpha)}\right)^{r}, \\
\theta_{2}(\alpha)=O(\alpha), \quad \theta_{2}(\alpha)=O\left(\alpha^{q / p}\right) .
\end{gathered}
$$

If we choose $\alpha=\alpha(\delta)=\delta^{p / \omega}$ with $\omega>1$, then we have

$$
\begin{gathered}
\delta\left(\frac{1}{\delta^{p / \omega}}\right)^{1 / p}=\delta^{(\omega-1) / w}, \\
\theta_{1}(\alpha)=O\left(\frac{1}{\log \left(1 / \delta^{p / \omega}\right)}\right)^{r}, \\
\theta_{2}(\alpha)=\delta^{p / \omega}, \quad \theta_{3}(\alpha)=O\left(\delta^{q / \omega}\right) .
\end{gathered}
$$

3.3. Example: Cauchy Problem for the Modified Helmholtz Equation. In this paragraph, we give a concrete example to see how to apply the theoretical results developed in this study.

Let us consider the Cauchy problem (modified Helmholtz equation) in the infinite strip $\mathbb{R} \times(0,1)$ :

$$
\begin{array}{r}
u_{y y}(x, y)+u_{x x}(x, y)-\gamma u(x, y)=0, \\
x \in \mathbb{R}, \quad y \in(0,1), \\
u(x, 0)=f(x), u_{y}(x, 0)=0, \quad x \in \mathbb{R},
\end{array}
$$

where $\gamma$ is a real positive constant.

Let $\widehat{u}(\xi, y)=(\mathscr{F} u)(\xi, y)$ be the Fourier transform of $u(x$, $y)$ :

$$
\widehat{u}(\xi, y)=\frac{1}{\sqrt{2 \pi}} \int_{\mathbb{R}} e^{-i \xi x} u(x, y) d x .
$$

With the help of the Fourier transformation, problem (1) can be transformed to an equivalent problem in the frequency domain:

$$
\begin{array}{r}
\widehat{u}_{y y}(\xi, y)-\xi^{2} \widehat{u}(\xi, y)-\gamma \widehat{u}(\xi, y)=0, \\
\xi \in \mathbb{R}, \quad y \in(0,1), \\
\widehat{u}(\xi, 0)=\widehat{f}(\xi), \widehat{u}_{y}(\xi, 0)=0, \quad \xi \in \mathbb{R} .
\end{array}
$$

It is easy to check that the formal solution of problem (92) has the form

$$
\widehat{u}(\xi, y)=\cosh \left(y \sqrt{\left(\xi^{2}+\gamma\right)}\right) \widehat{f}(\xi),
$$

or equivalently, the formal solution of problem (90) is given by

$$
\begin{aligned}
u(x, y) & =\left(\mathscr{F}^{-1} \widehat{u}\right)(x, y) \\
& =\frac{1}{\sqrt{2 \pi}} \int_{\mathbb{R}} e^{i x \xi} \widehat{u}(\xi, y) d \xi \\
& =\frac{1}{\sqrt{2 \pi}} \int_{\mathbb{R}} e^{i x \xi} \cosh \left(y \sqrt{\left(\xi^{2}+\gamma\right)}\right) \hat{f}(\xi) d \xi
\end{aligned}
$$

Putting $\Theta(\xi)=\sqrt{\left(\xi^{2}+\gamma\right)}$. Then $\Theta(\xi) \rightarrow+\infty$ as $|\xi| \rightarrow$ $+\infty$. From this remark, it is easy to see that a small perturbation in the data $\widehat{f}(\xi)$ may cause a dramatically large error in the solution $\widehat{u}(\xi, \xi)$. In addition, the magnifying factor is $\Theta(\xi) \sim e^{|\xi|}$, hence, the problem is severely ill-posed.

Since the data $f(\cdot)$ are based on (physical) observations and are not known with complete accuracy, we assume that $f$ and $f_{\delta}$ satisfy

$$
\left\|f-f_{\delta}\right\| \leq \delta
$$

where $f$ and $f_{\delta}$ belong to $L^{2}(\mathbb{R}), f_{\delta}$ denotes the measured data, and $\delta$ denotes the noise level.

For this problem, we define the regularized solutions with noisy data $f_{\delta}$ :

$$
\begin{aligned}
& u_{N}^{\delta}(x, y) \\
& \quad=\frac{1}{\sqrt{2 \pi}} \int_{\mathbb{R}} e^{i x \xi} \cosh \left(y \sqrt{\left(\xi^{2}+\gamma\right)}\right) \widehat{f}_{\delta}(\xi) \mathbf{1}_{[-N, N]}(\xi) d \xi \\
& \quad=\frac{1}{\sqrt{2 \pi}} \int_{-N}^{N} e^{i x \xi} \cosh \left(y \sqrt{\left(\xi^{2}+\gamma\right)}\right) \hat{f}_{\delta}(\xi) d \xi,
\end{aligned}
$$

where $\mathbf{1}_{[-N, N]}$ is the characteristic function of the interval $[-N, N]$. Consider

$$
\begin{aligned}
& u_{\alpha}^{\delta}(x, y) \\
& \quad=\frac{1}{\sqrt{2 \pi}} \int_{\mathbb{R}} e^{i x \xi}\left(\frac{\cosh \left(y \sqrt{\left(\xi^{2}+\gamma\right)}\right)}{1+\alpha e^{p \sqrt{\left(\xi^{2}+\gamma\right)}}}\right) \widehat{f}_{\delta}(\xi) d \xi,
\end{aligned}
$$

where $p \geq 1$. The quantities $\alpha=\alpha(\delta)$ and $N=N(\delta)$ are the parameters which were defined in Sections 3.1 and 3.2.

\section{The Nonlocal Boundary Value Problem Method and Some Extensions}

In this section we give the connection between the mollification method and the nonlocal boundary value problem method; also we give some extensions to our investigation. 
4.1. The Nonlocal Boundary Value Problem Method. Consider the following problem:

$P(a(a \geq 1) ; \alpha, \beta)\left\{\begin{array}{l}u_{y y}=A u, \quad 0<y<a L, \\ u(0)+\alpha u(a L)+\beta A^{-1 / 2} u_{y}(a L)=f, \\ u_{y}(0)=0 .\end{array}\right.$

(i) The case $\beta=0$ coincides with the method treated by Hào et al. in [10].

(ii) In the case $\alpha=\beta$, the solution of $P(a ; \alpha, \alpha)$ is $u_{\alpha}(y)=$ $\cosh (y \sqrt{A})\left(I+\alpha e^{a L}\right)^{-1} f$ coincides with the solution resulting from the mollification method.

(iii) The error estimates obtained in our analysis by using the mollification method are similar to those obtained in $[8,10]$.

This shows that our study framework is more general and includes many results obtained in this direction.

\subsection{Generalization. Let us consider}

$$
P\left\{\begin{array}{l}
u_{y y}=A u, \quad 0<y<L, \\
u(0)=f \\
u_{y}(0)=0
\end{array}\right.
$$

where $A$ is a self-adjoint, linear unbounded operator in $H$ and changes the sign with $0 \in \rho(A)\left(A^{-1}\right.$ exists and $\left.A^{-1} \in \mathscr{L}(H)\right)$.

We assume $\sigma(A)=]-\infty,-\gamma] \cup[\gamma,+\infty[, \gamma>0$. The spectral theory of self-adjoint operators enables us to write

$$
\begin{aligned}
h & =\int_{\mathbb{R}} d E_{\lambda} h=\int_{-\infty}^{-\gamma} d E_{\lambda} h+\int_{\gamma}^{+\infty} d E_{\lambda} h \\
& =h_{-}+h_{+}, \quad h \in H ;
\end{aligned}
$$

that is, the Hilbert space $H$ decomposes into the direct sum $H=H_{-} \oplus H_{+}$, and

$$
\begin{aligned}
A & =\int_{\mathbb{R}} \lambda d E_{\lambda} \\
& =\int_{-\infty}^{-\gamma} \lambda d E_{\lambda}+\int_{\gamma}^{+\infty} \lambda d E_{\lambda}=A_{-}+A_{+} .
\end{aligned}
$$

This decomposition gives us two problems: one is elliptic (ill-posed) and the other is hyperbolic (well-posed). Consider

$$
\text { Elliptic Problem }\left\{\begin{array}{l}
U y y=A_{+} U, \quad 0<y<L, \\
U(0)=f_{+}, \\
U_{y}(0)=0,
\end{array}\right.
$$

$$
\text { Hyperbolic Problem }\left\{\begin{array}{l}
V y y=A_{-} V, \quad 0<y<L, \\
V(0)=f_{-}, \\
V_{y}(0)=0 .
\end{array}\right.
$$

The formal solution of problem (99) is

$$
\begin{gathered}
u(y)=U(y)+V(y), \\
U(y)=\cosh \left(y \sqrt{A_{+}}\right) f_{+}, \\
V(y)=\cos \left(y \sqrt{-A_{-}}\right) f_{-} .
\end{gathered}
$$

We define

$$
\begin{aligned}
u_{\alpha}= & \cos \left(y \sqrt{-A_{-}}\right) f_{-} \\
& +\cosh \left(y \sqrt{A_{+}}\right)\left(I+\alpha e^{p L A_{+}}\right)^{-1} f_{+} \\
= & \int_{-\infty}^{-\gamma} \cos (y \sqrt{-\lambda}) d E_{\lambda} f \\
& +\int_{\gamma}^{+\infty} \cosh (y \sqrt{\lambda})\left(1+\alpha e^{p L \sqrt{\lambda}}\right)^{-1} d E_{\lambda} f, \\
v_{\alpha}= & \cos \left(y \sqrt{-A_{-}}\right) f_{-}+\cosh \left(y \sqrt{A_{+}}\right) E_{\beta} f_{+} \\
= & \int_{-\infty}^{-\gamma} \cos (y \sqrt{-\lambda}) d E_{\lambda} f \\
& +\int_{\gamma}^{+\infty} \cosh (y \sqrt{\lambda}) \mathbf{1}_{[\gamma, \beta]} d E_{\lambda} f .
\end{aligned}
$$

We follow the same methodology developed in the previous Sections 3.1 and 3.2, we show that $u_{\alpha}$ and $v_{\alpha}$ are two stable approximations to problem (99), and we establish the same results of error estimates.

Remark 26. We define the mollification operator $M_{\alpha}=(I+$ $\left.\alpha \Theta_{p}(A)\right)^{-1}$, where $\Theta_{p}:[\gamma,+\infty[\rightarrow \infty$ satisfies

$$
\forall \lambda \in\left[\gamma,+\infty\left[, \quad \Theta_{p}(\lambda) \geq e^{p L \sqrt{\lambda}}, \quad p \geq 1 .\right.\right.
$$

Under certain conditions on $\Theta_{p}(\cdot)$ and with the help of a technical calculation, we can extend the results obtained in Section 3.2.

\section{Acknowledgments}

The authors are grateful to the editor and the anonymous referees for their valuable comments and helpful suggestions which have much improved the presentation of the paper. This work is supported by the MESRS of Algeria (CNEPRU Project B01120090003).

\section{References}

[1] A. Qian, J. Mao, and L. Liu, "A spectral regularization method for a Cauchy problem of the modified Helmholtz equation," Boundary Value Problems, vol. 2010, Article ID 212056, 13 pages, 2010.

[2] G. Alessandrini, "Stable determination of a crack from boundary measurements," Proceedings of the Royal Society of Edinburgh A, vol. 123, no. 3, pp. 497-516, 1993. 
[3] D. D. Ang, R. Gorenflo, V. K. Le, and D. D. Trong, Moment Theory and Some Inverse Problems in Potential Theory and Heat Conduction, vol. 1792 of Lecture Notes in Mathematics, Springer, Berlin, Germany, 2002.

[4] A. M. Denisov, E. V. Zakharov, A. V. Kalinin, and V. V. Kalinin, "Numerical solution of the inverse electrocardiography problem with the use of the Tikhonov regularization method," Computational Mathematics and Cybernetics, vol. 32, no. 2, pp. 61-68, 2008.

[5] M. M. Lavrentev, V. G. Romanov, and G. P. Shishatskii, IllPosed Problems in Mathematical Physics and Analysis, American Mathematical Society, Providence, RI, USA, 1986.

[6] L. E. Payne, Improperly Posed Problems in Partial Differential Equations, Society for Industrial and Applied Mathematics, Philadelphia, Pa, USA, 1975.

[7] G. Alessandrini, L. Rondi, E. Rosset, and S. Vessella, "The stability for the Cauchy problem for elliptic equations," Inverse Problems, vol. 25, no. 12, Article ID 123004, 47 pages, 2009.

[8] U. Tautenhahn, "Optimal stable solution of Cauchy problems for elliptic equations," Zeitschrift für Analysis und ihre Anwendungen, vol. 15, no. 4, pp. 961-984, 1996.

[9] Z. Qian, C.-L. Fu, and Z.-P. Li, “Two regularization methods for a Cauchy problem for the Laplace equation," Journal of Mathematical Analysis and Applications, vol. 338, no. 1, pp. 479489, 2008.

[10] D. N. Hào, N. V. Duc, and D. Lesnic, "A non-local boundary value problem method for the Cauchy problem for elliptic equations," Inverse Problems, vol. 25, no. 5, Article ID 055002, 27 pages, 2009.

[11] X.-L. Feng, L. Eldén, and C.-L. Fu, "A quasi-boundary-value method for the Cauchy problem for elliptic equations with nonhomogeneous Neumann data," Journal of Inverse and IllPosed Problems, vol. 18, no. 6, pp. 617-645, 2010.

[12] I. V. Mel'nikova, "Regularization of ill-posed differential problems," Siberian Mathematical Journal, vol. 33, no. 2, pp. 289-298, 1992.

[13] P. N. Vabishchevich and A. Yu. Denisenko, "Regularization of nonstationary problems for elliptic equations," Journal of Engineering Physics and Thermophysics, vol. 65, no. 6, pp. 11951199, 1993.

[14] V. A. Kozlov and V. G. Maz'ya, "On iterative procedure for solving ill-posed boundary value problems that preserve differential equations," Leningrad Mathematical Journal, vol. 1, no. 5, pp. 1207-1228, 1990.

[15] D. N. Hào, "A mollification method for ill-posed problems," Numerische Mathematik, vol. 68, no. 4, pp. 469-506, 1994.

[16] N. Dunford and J. Schwartz, Linear Operators-Part II, John Wiley \& Sons, New York, NY, USA, 1967.

[17] A. Pazy, Semigroups of Linear Operators and Applications to Partial Differential Equations, vol. 44 of Applied Mathematical Sciences, Springer, New York, NY, USA, 1983.

[18] S. G. Kreĭn and Ju. I. Petunin, "Scales of Banach spaces," Uspekhi Matematicheskikh Nauk, vol. 21, no. 2(128), pp. 89-168, 1966.

[19] H. O. Fattorini, The Cauchy Problem, vol. 18 of Encyclopedia of Mathematics and Its Applications, Addison-Wesley Publishing, Reading, Mass, USA, 1983.

[20] K. Schmüdgen, Unbounded Self-Adjoint Operators on Hilbert Space, vol. 265 of Graduate Texts in Mathematics, Springer, Dordrecht, The Netherlands, 2012. 


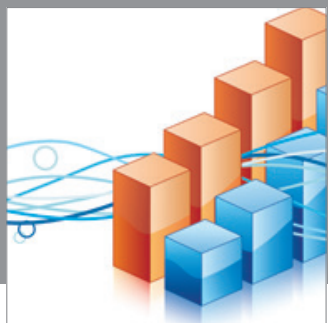

Advances in

Operations Research

mansans

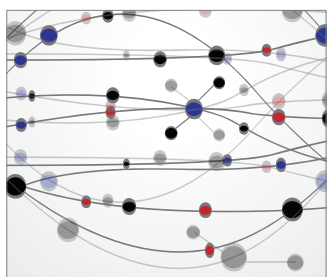

The Scientific World Journal
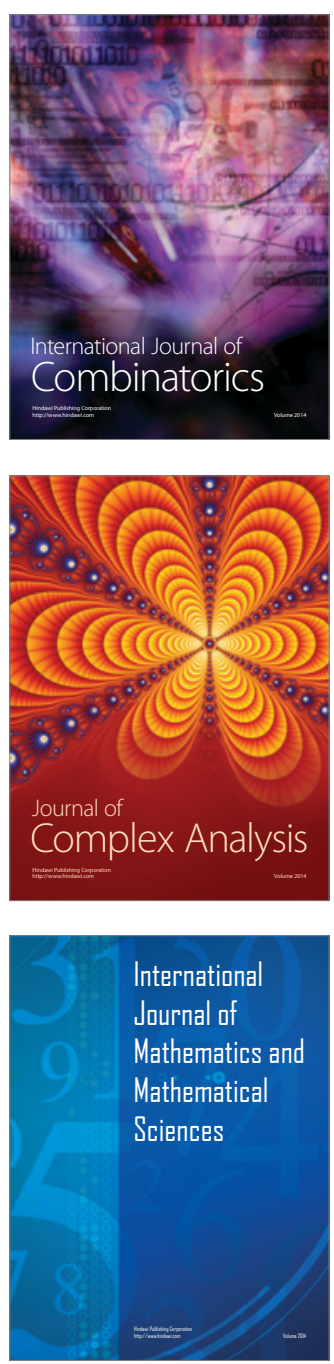
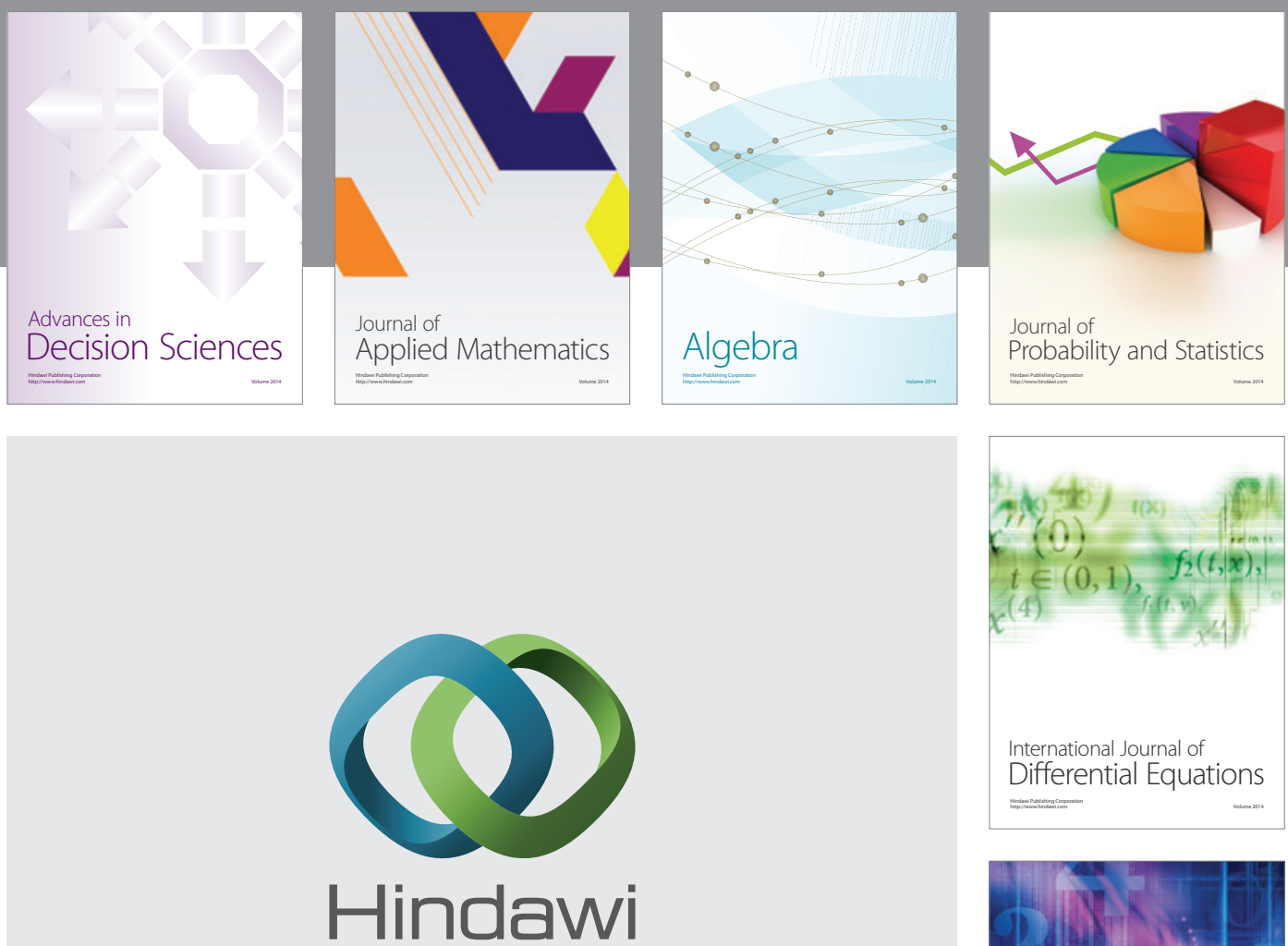

Submit your manuscripts at http://www.hindawi.com
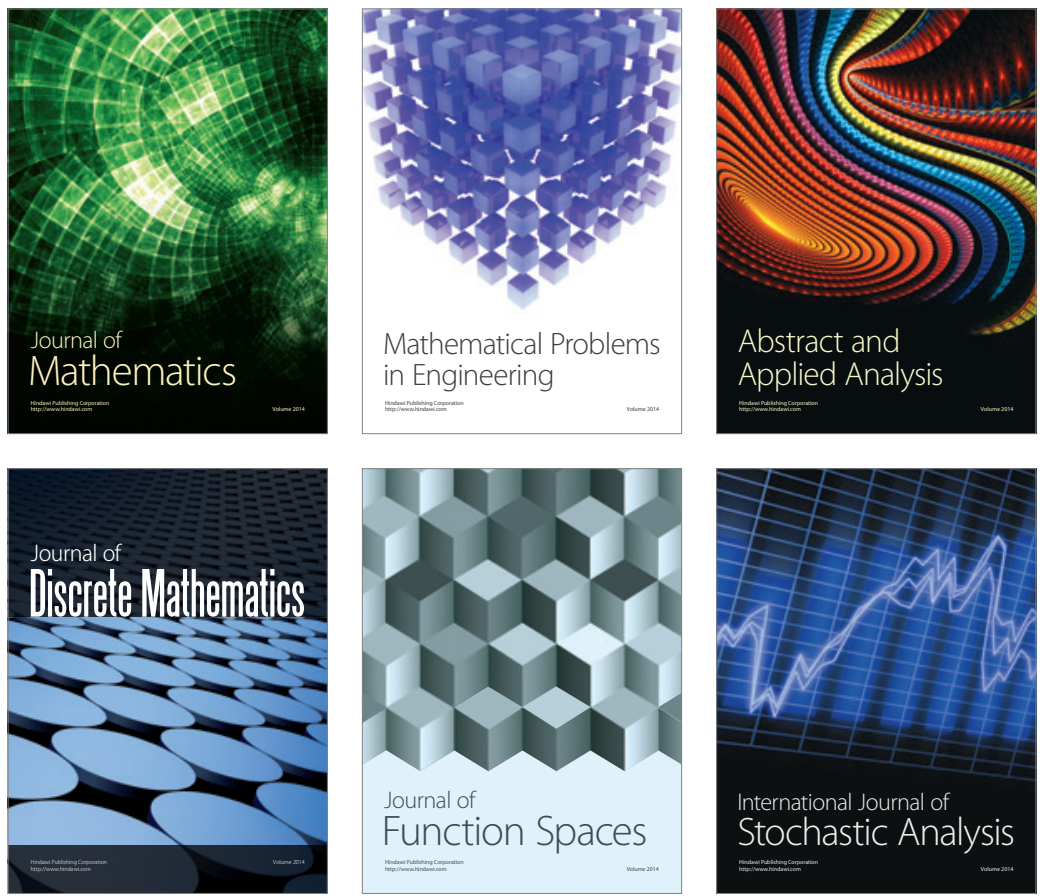

Journal of

Function Spaces

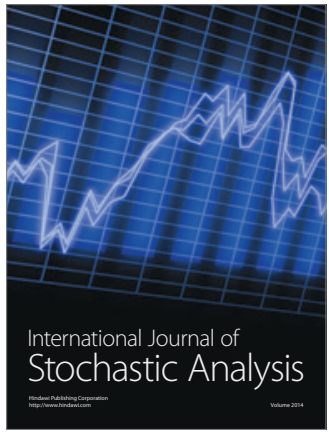

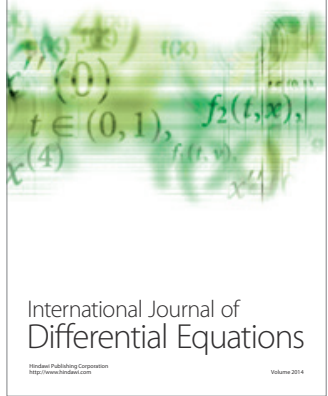
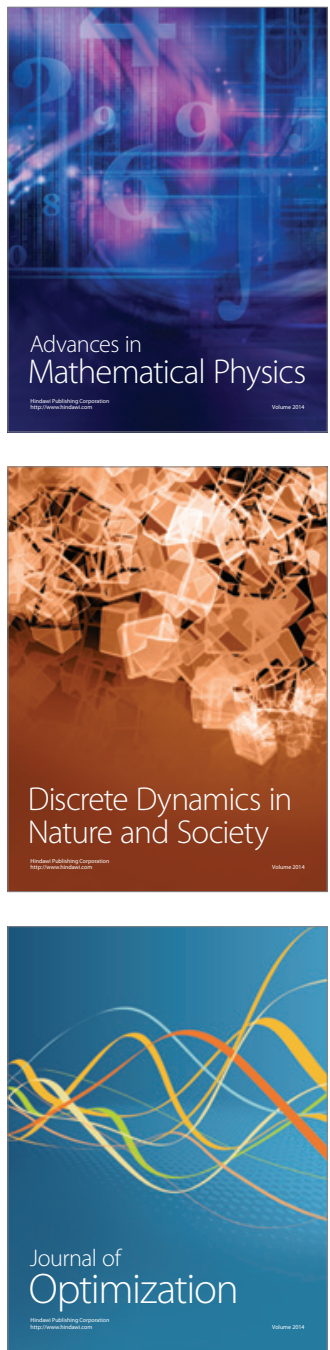